\title{
Altered Disease Risk from Climate Change
}

Today's global climate crisis poses large risks to public health through many exposure pathways; from heat waves and air pollution, to infectious diseases, malnutrition, and social dislocation. Climate change has the potential to reverse many of the recent gains seen in the reduction in infectious diseases globally, while exacerbating the challenge of health inequities (Woodward et al. 2014).

This special issue of EcoHealth contains new studies that illustrate the sensitivity of health outcomes to a changing climate, as well as opportunities for predicting risk and thereby improving the effectiveness of early interventions. These new studies add to the growing literature linking climate change to public health. Since the establishment of the United Nations Intergovernmental Panel on Climate Change (IPCC) 30 years ago to assess climate change science, impacts and mitigation, it is clear that health concerns are now central to the climate change discourse.

Addressing the more direct consequences of exposure to excessive heat, Limaye et al. (2018) estimate that in the year 2069, the eastern USA could experience over 11,000 additional deaths due to projected increases in apparent summer temperatures of more than $4^{\circ} \mathrm{C}$. But concern about climate change extends beyond the direct effect of elevated temperatures, as bat-borne and mosquito-borne diseases are more sensitive to subtle shifts in weather conditions and local ecology. In two papers by Martin et al. (2018a, b), the authors argue that Hendra virus may spread southward due to shifts in climate-related habitat suitability for the fruit bat reservoir species. The authors find a subsequent doubling in the number of horses at risk from Hendra virus infection. They further investigate patterns of future climate over the Australian landscape and posit that weather-induced food shortages could alter bat immune systems or behavior, or potentially adaptive responses in horse husbandry.

Disease prevention occurs at several levels along the exposure-response continuum, and the earlier that detection can occur, the more likely interventions will be successful. In this issue, Wollenberg Valero et al. (2017) examine how changing climate may alter plant phenology for predicting the likelihood of Ebola virus spillover events in Africa. The authors conclude that plant phenology-as an integrated biologic response to multiple weather parameters-may be a useful predictor of Ebola spillover events. In the USA, Egizi et al. (2018) use mosquito blood meals to assess circulating pathogens in a Culex mosquito species that can carry arboviruses, as a way to monitor the expansion of the geographic range in disease risk.

The rationale for actions to mitigate climate change also takes on ethical dimensions, as we first discussed in a 2007 issue of EcoHealth, showing that populations most vulnerable to health risks from climate change are those least responsible for causing the problem (Patz 2007). In this issue, an editorial by Frumkin and Watts (2018) provides an update on the ethics of climate change, expanding on foundational recommendations from Pope Francis' sweeping 2015 encyclical Laudato Si, "On Care of our Common Home."

For health and for ethical reasons, the global climate crisis demands rapid collective actions to divert our current path toward a $7^{\circ} \mathrm{C}$ warmer world by the end of this century. But consider the fact that fossil fuel combustion accounts for the majority of greenhouse gas emissions-with deforestation comprising between a quarter and a third of emissions due to the loss of the carbon sink that forests provide. Cleaner energy can help reduce the heating of the planet, while saving lives from air pollution. In addition, 
upward trends in obesity and chronic diseases, such as diabetes and heart disease, are now occurring throughout the world, as Western lifestyles with automobile-dependent transportation and meat-based diets are being pursued. Herein lie substantial opportunities for public health by: First, adopting more alternative modes of transportation, especially those that promote exercise through "active transport" by foot or by bicycle; and second, reducing meat in the diet (Patz et al. 2014).

In conclusion, disease risks posed by climate change must be evaluated alongside the health benefits likely to accrue from a low-carbon economy and forest protection. Such an approach requires a more diverse set of indicators to measure progress across many sectors of society, in addition to disease monitoring and conventional risk assessment (Watts et al. 2018). In parallel, we continue to need better understanding of how a changing climate will alter disease dynamics and pose risks to health at the local level.

Jonathan A. Patz

Global Health Institute,

University of Wisconsin, 1710 University Avenue, Madison, WI 53726, USA

e-mail: patz@wisc.edu

\section{REFERENCES}

Egizi A, Martinsen ES, Vuong H, et al. (2018) Using bloodmeal analysis to assess disease risk to wildlife at the new northern limit of a mosquito species. EcoHealth 15(3):543-554. https:// doi.org/10.1007/s10393-018-1371-0

Frumkin H, Watts N (2018) Health, science, faith, and stewardship. EcoHealth 15(3):482-484. https://doi.org/10.1007/s10393018-1373-y

Limaye VS, Vargo J, Harkey M, et al. (2018) Climate change and heat-related excess mortality in the Eastern USA. EcoHealth 15(3):485-496. https://doi.org/10.1007/s10393-018-1363-0

Martin G, Yanez-Arenas C, Chen C, et al. (2018a) Climate change could increase the geographic extent of Hendra virus spillover risk. EcoHealth 15(3):509-525. https://doi.org/10.1007/s10393018-1322-9

Martin G, Yanez-Arenas C, Plowright RK, et al. (2018b) Hendra virus spillover is a bimodal system driven by climatic factors. EcoHealth 15(3):526-542. https://doi.org/10.1007/s10393-0171309-y

Patz JA, et al. (2014) Climate change: challenges and opportunities for global health. JAMA 312(15):1565-1580

Patz JA, Gibbs HK, Foley JA, Rogers JV, Smith KR (2007). Climate change and global health: quantifying a growing ethical crisis. EcoHealth 4(4):397-405. https://doi.org/10.1007/s10393-0171309-y

Watts N, et al. (2018) The Lancet Countdown on health and climate change: from 25 years of inaction to a global transformation for public health. Lancet 391(10120):581-630

Wollenberg Valero KC, Isokpehi RD, Douglas NE, et al. (2018) Plant phenology supports the multi-emergence hypothesis for Ebola spillover events. EcoHealth 15(3):497-508. https://doi.org/ 10.1007/s10393-017-1288-Z

Woodward A, et al. (2014) Climate change and health: on the latest IPCC report. Lancet 383(9924):1185-1189

Published online: October 30, 2018 